Case Report

\title{
BLACK HIP-A CASE OF OCHRONOTIC ARTHROPATHY
}

\author{
Lawrence J. Mathias ${ }^{1}$, Michelle Mathias ${ }^{2}$, Padma Shetty ${ }^{2}$, Ravindranath Rai ${ }^{1}$ \\ ${ }^{1}$ Department of Orthopedics, ${ }^{2}$ Department of Pathology, K.S Hegde Hospital, Nitte University, M angalore, India \\ Correspondence : \\ Lawrence Mathias \\ Dept of Orthopedics, K.S. Hegde Charitable Hospital, Mangalore - 575018 \\ Mobile : 98454 51040, E-mail : larrymathias@yahoo.co.in
}

\begin{abstract}
:
alkaptonuria an autosomal recessive disorder in which a deficiency of homogentessic acid oxidase results in the accumulation of homogentessic acid which is deposited in connective tissues notably in the intervertebal disc,articular cartilage and tendons leading to their early degeneration. Here we describe a case of ochronotic arthropathy
\end{abstract}

Keywords: Alkaptonuria, ,Ochronotic arthropathy

\section{Introduction:}

The musculoskeletal manifestation of alkatonuria is called "Ochronosis". A term coined by Virchow because of the Ochre or yellow appearance of cartilage under the microscope. By the fourth or fifth decade the diseases progresses from simple alkaptonuria to alkaptonuric arthropathy in approximately $30 \%$ of subjects .

\section{Case Report :}

A 36 year old male presented, with pain in his groin with difficulty in walking. The movements of the hip were markedly painful and restricted. Radiographs showed a decrease in joint space with subchondral sclerosis. An excision arthroplasty was done,considering his social and economic status. Macroscopically brownish black pigmentation was found in the cartilage of femoral head. (fig2)

Histopathologically the tissue showed degenerative change such as eburnation and atrophy of the subchondral bone of weight bearing area and yellowish pigmentation of the articular cartilage (fig 1 ). In addition to these findings it was noted that there was a migration of the destructed articular cartilage down to the subchondral lesion which was surrounded by macrophages. In the non weight bearing area, cartilage still remained in the joint with chondrocytic cloning. Fragmentation and fibrillation of cartilage were increased in the boundary area which was clearly distinct between weight bearing and non weight bearinglesions.
Retrospectively radiographs of the spine revealed the characteristic appearance of Ochronosis with narrowing of disc spaces and calcification and sclerosis of vertebral margin (fig3).The urine changed color to dark brownish hew on standing for a few hours and tested positive for homogentessic acid. Histopathological examination showed deposition of yellow to dark pigment in the fibrocollagenous tissue. The patient had no previous symptoms related to Ochronosis.

\section{Discussion :}

Alkaptonuria (urine which darkens on exposure to air) is a rare hereditary metabolic disorder with an incidence of 1:1000000 individuals. It is transmitted as an autosomal necessive disorder affecting males and females equally,in which the HGO gene 1coding for homogentessic oxidase an enzyme found in liver and kidney are absent which catalyses Homgentessic acid(HGA) to maleylacetoacetic acid. $^{2}$

The patients are usually asymptomatic until early adulthood pigmentation of the sclera or cartilage of the ear may appear by the second decade and this may also be seen in the teeth, buccal mucosa and in the nails of skin giving these areas a dusty color. The clinical manifestations are caused by the accumulation of homogentestic acid in the fibrillary collagens of connective tissue to which it binds irreversibily by polymerization and oxidation The accumulation of HGA modifies the quality of the cartilage that becomes friable and degenerates.Under 
electronmicroscope the articular cartilage shows erosion of the surface,pigment accumulation in the chondrocyte and intercellular matrix,chondrocytic degeneration. Alkaptonuric arthropathy ,the only disabling effect of the disease is due to stress and fragmentation of the articular

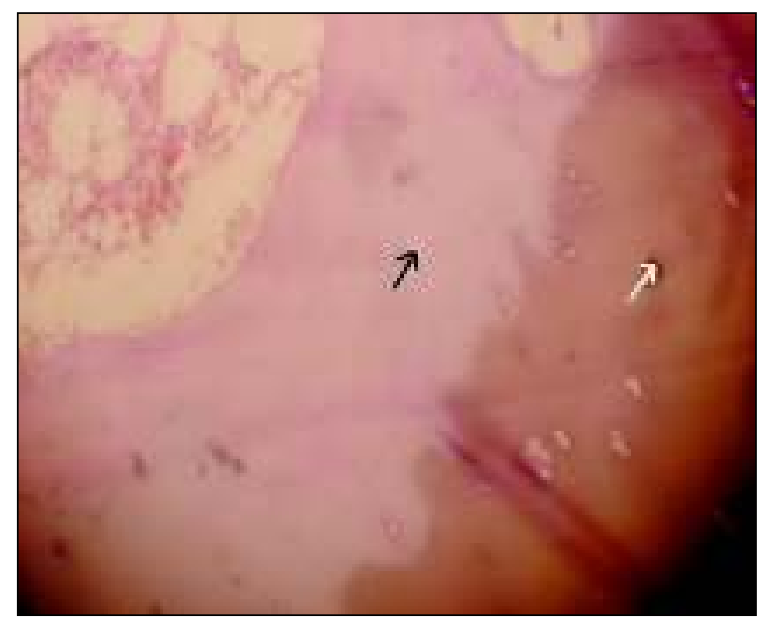

cartilage showing ochre pigment(white arrow), with underlying bone(black arrow), marrow elements and fat spaces.[ H\&E stain $\times 100 x]$

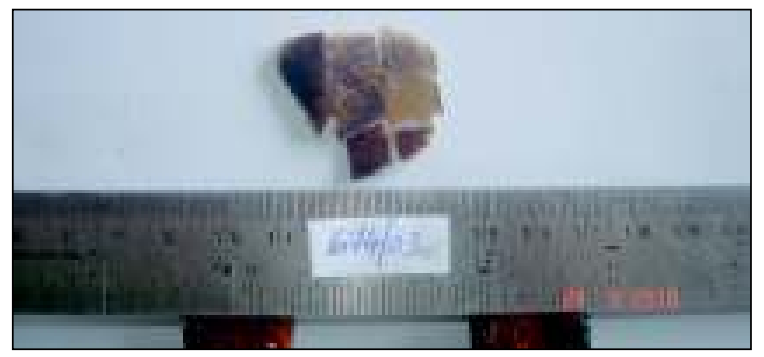

cut surface of head of femur showing black pigmentation

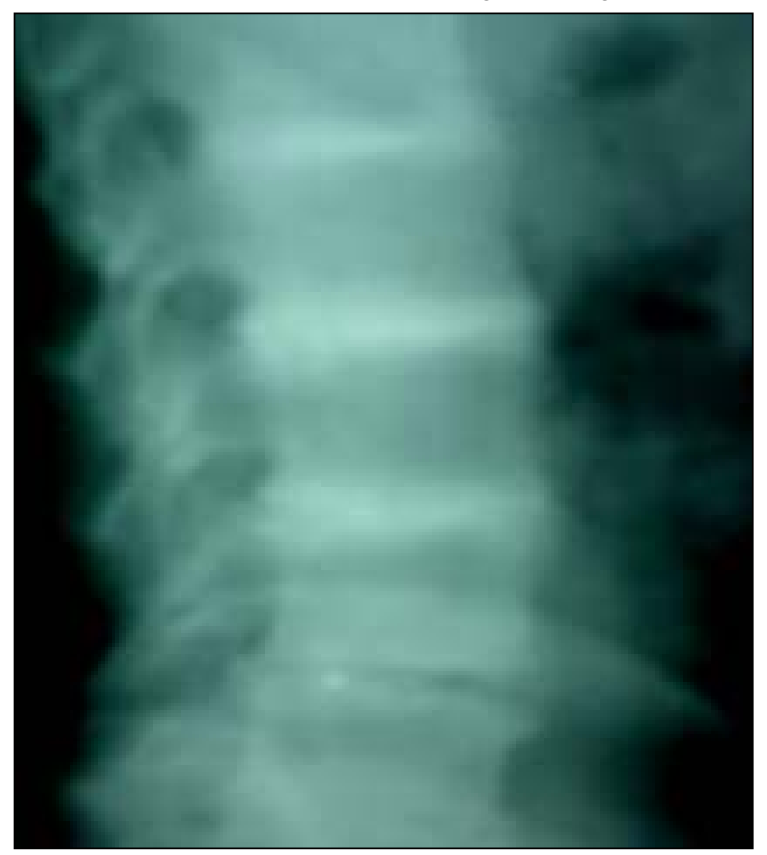

$x$-ray showing calcification of the intervertebral disc cartilage . The spine and major weight bearing joints are most commonly affected and symptoms start by the fourth decade,with the changes occurring more rapidly in men. The hip bones and other weight bearing joints are often involved and the changes may be severe enough to suggest arthroplasty, which in our case we did an excision arthroplasty for socio-economic reasons.

In ochronotic spondylosis the entire spine is affected, symptoms are mainly related to the lumbar region. Extensive calcification of the intervertebral discs which appear elliptical and opaque is very characteristic.

The numerous reports on alkaptonuria are limited to degenerative manifestations in joints. There are only a few reports of effects of the deposition of homogetesic acid in tendon. Homogentesic Acid is widely deposited in connective tissue and such pigmentation must include tendons which contain type I collagen. The accumulation of homogentesic acid inhibits collagen cross limiting leading to reduction of the structural integrity of the collagen , thus increasing the likelyhood of spontaneous rupture.

There is no specific treatment for alkatonuria . Reduction of tyrosine and phenylalamine in the diet has been reported to reduce the excretion of homogentisate. It is not known whether dietary restrictions from early life would avoid or minimize later complications but such an approach is reasonable for older children and adults. Nitisinone an experimental drug, inhibits the enzyme that produces HGA and leads to a reduction in the urinary HGA excretion .

\section{References:}

1. Phornphutkul C, Introne WJ, Perry MB, Bernardini I, Murphey MD, Fitzpatrick DL, Anderson PD, Huizing M , Anikster Y, Gerber LH, Gahl WA Natural history of alkaptonuria. N Engl J M ed 2002;347:2111-21.

2. Turgay E, Canat D, Gurel MS, Yuksel T, Baran MF, Demirkesen C Endogenous ochronosis. Clin Exp Dermatol 2009;34:e865-e868.

3. Di FM , Coari G, Bonucci E A morphological study of bone and articular cartilage in ochronosis. Virchows Arch 2000;436:74-81.

4. Iannuccelli C, Coari G, Mastantuono M, Osimani M, Valesini G, Di FM Joint and tendineous involvement in ochronosis: clinical history and imaging of a case. Clin Exp Rheumatol 2009;27:696-7.

5. Jebaraj I, Rao A Achilles tendon enthesopathy in ochronosis. J Postgrad Med 2006;52:47-8.

6. MacKenzie $C R$, Major $P$, Hunter $T$ Tendon involvement in a case of ochronosis. J Rheumatol 1982;9:634-6. 